\title{
RECENT DEVELOPMENTS IN IMPROVING DAYTIME ANGULAR RESOLUTION FROM THE GROUND
}

\author{
K. O. KIEPENHEUER \\ Fraunhofer Institut, Freiburg, F.R.G.
}

The subject of this symposium is the fine structure of the solar chromosphere. Progress in this field of reserach will depend to a high degree of the quality of seeing, resp. on the effective angular resolution available on the ground. Today's situation of solar ground seeing has changed distinctly in the last years. I would like to report here a few new aspects, which could be condensed into 3 questions:

(1) Are there on the ground 'good seeing windows', comparable in quality with stratosphere results obtained from balloon borne equipment?

(2) Is there a chance to resolve from the ground the solar scale height, corresponding to about $0.1^{\prime \prime}$.

(3) Is there a residual fundamental atmospheric seeing noise resp. a basic limit to the atmospheric Modulation Transfer Function (MTF)?

Within the European JOSO project (Joint Organization for Solar Observation) we have tried hard to answer these questions. Quite on purpose we deferred for the present the construction of a joint observatory and concentrated on the systematic investigation of our own atmosphere and the search for an optimal site. It is clear: as soon as an observatory site has been agreed upon this kind of activity, somewhat unusual for an astronomer, will stop.

I will leave out here the mistakes and detours which we made in this field which was new to us. One thing which we have learned is that to move around on the ground with an appropriate telescope is the most uneconomic way to find a site. This telescope would have to be put at each testing point on a very rigid and expensive tower of some $20 \mathrm{~m}$ height. And this telescope would give only the integrated effect of the atmospheric disturbances all along the line of sight. Instead we aimed first to investigate the atmosphere above promising areas directly by recording the inhomogeneities 'in situ' in the form of temperature (resp. density) fluctuations.

The measurements have been made from high masts, from tethered and from free flying balloons (with radiosonde up to $15000 \mathrm{~m}$ ) and from slow flying aircrafts (up to $5000 \mathrm{~m}$ ). Temperature fluctuations down to $0.01 \mathrm{~K}$ with a time resolution of better than $0.002 \mathrm{~s}$ could be recorded. This corresponds for an aircraft flying with $150 \mathrm{~km} \mathrm{~h}^{-1}$ to a spatial resolution of better than $10 \mathrm{~cm}$.

Here are our main results:

(1) We could prove quantitatively the high degree of homogeneity of the atlantic airmass as compared to the airmass over the continent by aircraft scans. The difference of the two is most striking in altitudes below $3000 \mathrm{~m}$. By means of balloon-borne radiosondes for recording temperature fluctuations up to about $18000 \mathrm{~m}$ altitude, it was 
furthermore shown that the contribution of the upper troposphere and of the tropopause is relativily small or even negligible, at least above the southern atlantic ocean within the high pressure system of the Azores and under fairly stable weather conditions.

Under certain assumptions (isotropic turbulence, validity of the Kolmogoroff law) one can - following J. E. Coulman (1969) - calculate the Modulation Transfer Function (MTF) of an atmosphere with given temperature fluctuations. Based on the aircraft and balloon records obtained over the Canary islands we found quite often that the free airmass above ca. $3000 \mathrm{~m}$ (troposphere and tropopause) would yield an MTF of ca. $40 \%$ for an angular separation of $0.1^{\prime \prime}$ ? This implies of course that the telescope can be brought in direct contact with this airmass.

(2) The direct optical access to this exceptional airmass resp. to its MTF is the more difficult problem: Usually there will be a convective or a turbulent layer of several hundred up to about $2000 \mathrm{~m}$ thickness above ground. The only places which we found and proved to be suitable were certain mountain sites in the atlantic, which because of their specific topography showed a laminar flow of air down to $<30 \mathrm{~m}$ above ground, at least for a certain range of windspeed. It is this laminar flow which brings the undisturbed airmass close to the site. In addition it wipes off the local turbulence and keeps it away from the line of sight. This situation we found on the Pico de Teide $(3717 \mathrm{~m})$ on the island of Tenerife, some $600 \mathrm{~km}$ away from the African W-coast, with its very regular conical shape, and on the Muchachos $(2430 \mathrm{~m})$, highest point of the island of La Palma, showing very smooth slopes to NW and W, which are the prevailing wind directions at this altitude. Figures 1 and 2 give some typical records of temperature fluctuations obtained above the two sites for various windspeeds. They show clearly, that already $30 \mathrm{~m}$ above the sites the fluctuations can be as small as a few hundredths of a degree, if the wind speed exceeds ca. $6 \mathrm{~m} \mathrm{~s}^{-1}$, while for calm days with wind speed $<4 \mathrm{~m} \mathrm{~s}^{-1}$ the local convection forms a turbulent layer of a few hundred meters thickness above the site.

No doubt, there are many other mountains of this height, possibly even with better aerodynamic properties, but obviously there are extremely few being exposed continuously to this kind of stable and homogeneous airmass as in the case of the Canary islands. The Teide site will certainly give more hours of excellent seeing for a given height of the telescope above ground, than the Muchachos site. But it will produce more technical and logistic problems because of its height and its small size. It will be the task of JOSO - after having conducted the final optical test - to come to a reasonable compromise between these two sites.

(3) Altogether we have learned that the absolute altitude of a site is more important than we had expected. Apart from disturbances near to the ground tropospheric turbulence seems to occur mostly in the form of relatively thin layers, most of them appearing below $3000 \mathrm{~m}$ altitude. Sea level or low level sites, whether above the ocean or above the continent, will therefore probably reach the quality of a site above 2000 or $3000 \mathrm{~m}$, only for short moments. We are nevertheless still investigating a sea level site in southern Portugal (Utrecht Group). 


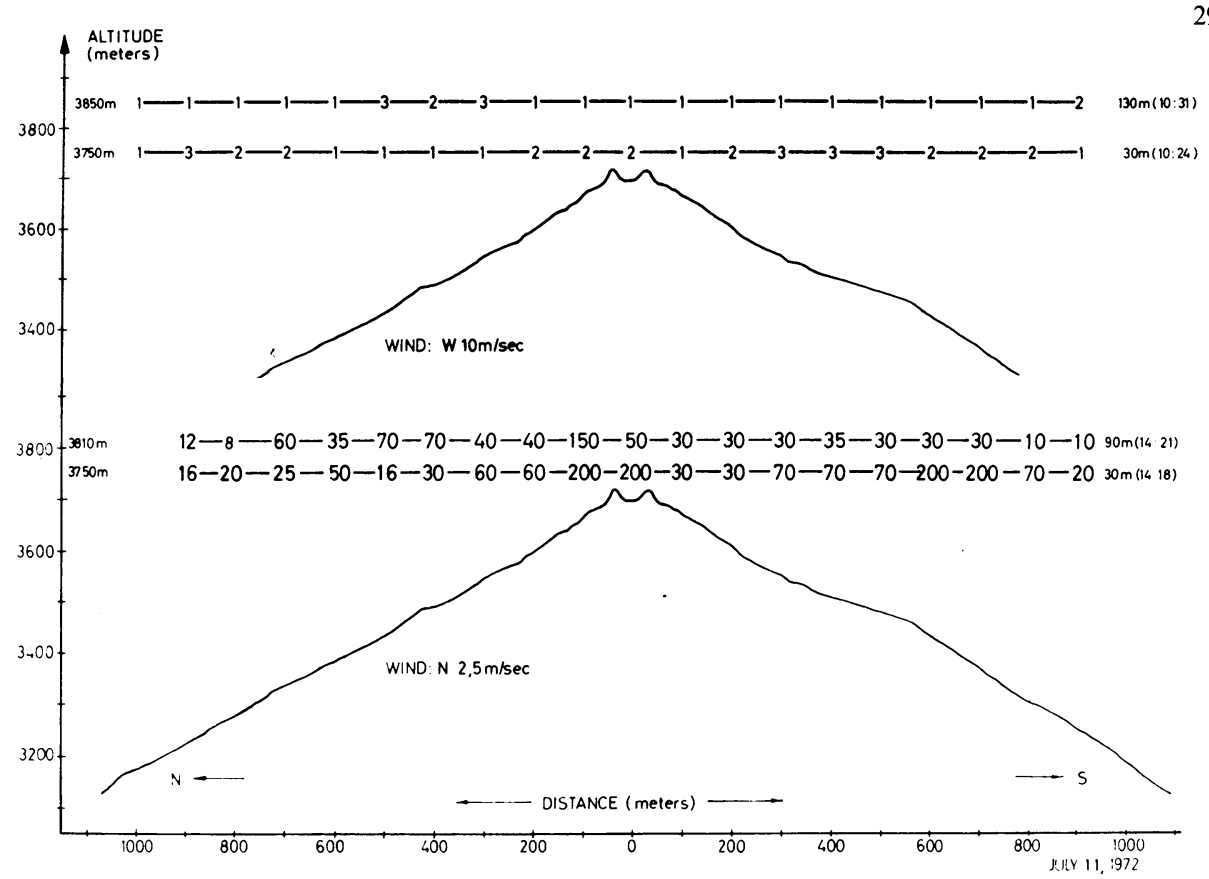

Fig. 1. Temperature fluctuations recorded by aircraft above the Pico de Teide $(3718 \mathrm{~m})$ in units of $0.01 \mathrm{~K}$. Upper record: 1972, July 15 , windspeed $12.5 \mathrm{~m} \mathrm{~s}^{-1}$; below 1972 , July 11 , windspeed $5 \mathrm{~m} \mathrm{~s}^{-1}$.
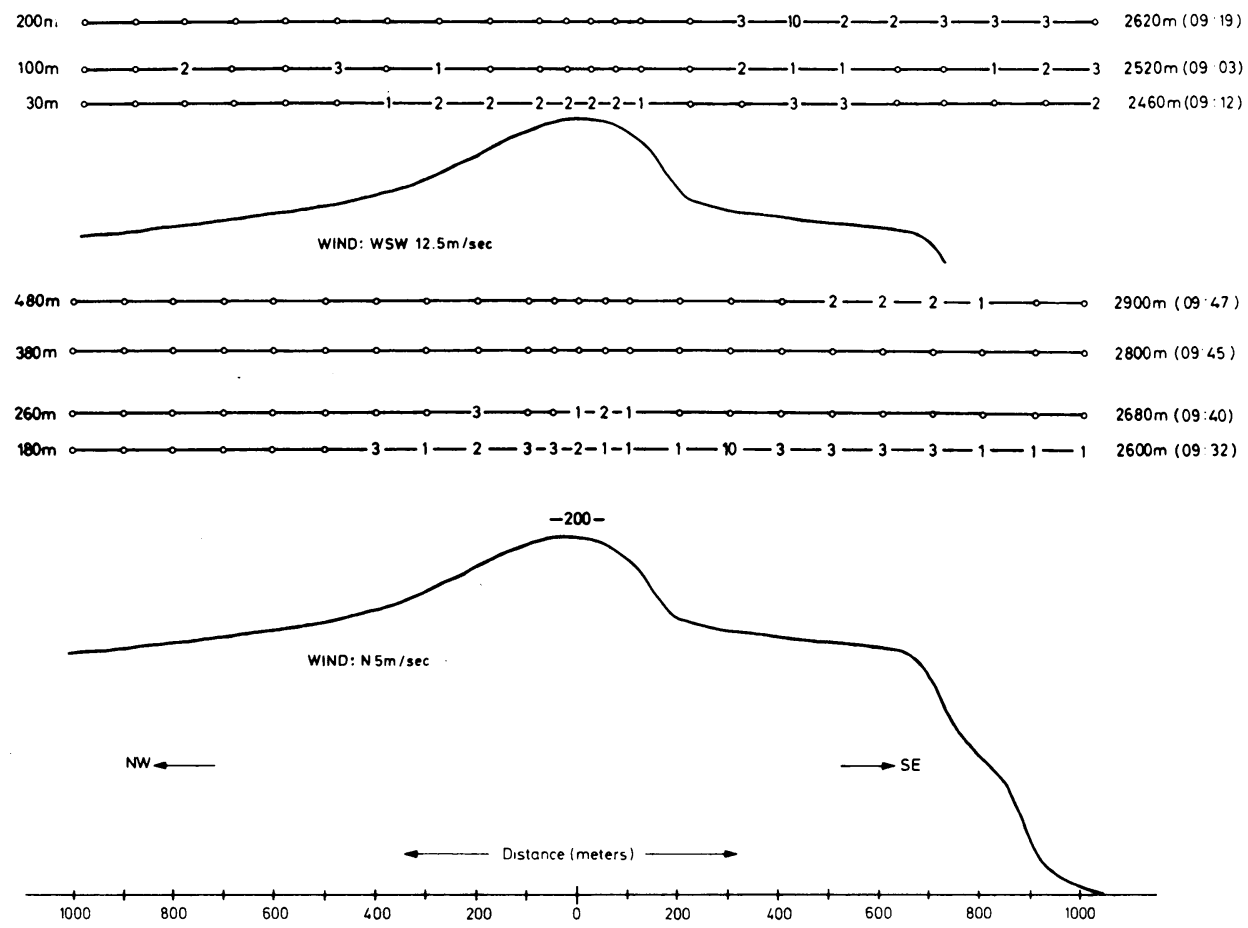

Fig. 2. Temperature fluctuation recorded by aircraft above Roque de los Muchachos $(2430 \mathrm{~m})$ in units of $0.01 \mathrm{~K}$. Upper record: 1973 , June 20 , windspeed $10 \mathrm{~m} \mathrm{~s}^{-1}$; below 1973 , June 20 , windspeed $5 \mathrm{~m} \mathrm{~s}^{-1}$. The value of $200(=2 \mathrm{~K})$ just above the summit was obtained on a flight perpendicular to the drawing plane. 
With this information in hand, we can now try to answer provisionally the three questions which I presented initially:

There are indeed large 'windows' of excellent seeing in our atmosphere. They occur above $2000 \mathrm{~m}$ altitude above the ocean and will guarantee for long periods an image quality as obtained with balloon-borne equipment in the stratosphere, probably even better than this, if larger instruments can be used. These large 'windows', however, shrink to bull's eyes if a direct optical access to the stable airmass on the ground or in buildable heights above the ground is wanted. Because then the complicated condition of laminar airflow and absence (or wiping off) of the thermal convection has to be fullfilled. Indeed, such windows are extremely rare. But they do exist and the corresponding sites will very probably enable us to resolve the solar scale height from the ground in pictures as well as in long exposure spectrograms during long periods of time.

The residual inhomogeneities which we could trace even high above the ocean in a stable airmass (Clear Air Turbulence) by aircraft and by free flying balloons turned out to be mostly so small that their optical effects along the line of sight will on the average be important only for very large solar telescopes (aperture $\gtrsim 150 \mathrm{~cm}$ ).

These results could not have been obtained without the fortunate and stimulating cooperation of solar astronomers of all European countries which devoted a considerable part of their time to the JOSO project. The two German aircraft campaigns (Fraunhofer Institut, Freiburg) were kindly sponsored by the Deutsche Forschungsgemeinschaft in Bonn and by the Institut National d'Astronomie et de Géophysique in Paris. The Italian radio sonde campaign in Tenerife (Arcetri and Catania observatories) was sponsored by the Consiglio Nacionale delle Richerche, Rome.

Further details can be found in the JOSO Reports SIT 15 and SIT 17 as well as in the Annual JOSO Reports for 1970, 1971 and 1972 and in the forthcoming Italian JOSO Report on the radiosonde campaign 1973.

\section{Reference}

Coulman, J. E.: 1969, Solar Phys. 7, 122.

\section{DISCUSSION}

Jefferies commented that the same favorable conditions apply to the mountain observatories in the Hawaiian Islands. Kiepenheuer agrees, except that the conditions there are not quite as stable because of the larger extent of the mountains. The critical point is therefore higher up so that one probably has to go higher above the ground to reach the steady flow.

The sunshine record is $95 \%$ for the Teide, a little less at the site on La Palma (this in reply to a question by Giovanelli). 